\title{
A NECESSARY AND SUFFICIENT CONDITION FOR THE EXISTENCE Of A UNIQUE SOLUTION OF A DISCRETE BOUNDARY VALUE PROBLEM
}

\author{
RAGHIB ABU-SARIS and WAJDI AHMAD
}

Received 4 November 2002 and in revised form 25 February 2003

\begin{abstract}
A $k$ th-order linear difference equation with constant coefficients subject to boundary conditions is considered. A necessary and sufficient condition for the existence of a unique solution for such a boundary value problem is established. The condition established answers a fundamental question for well-posedness and can be easily applied using a simple and computationally tractable algorithm that does not require finding the roots of the associated characteristic equation.
\end{abstract}

2000 Mathematics Subject Classification: 39A10.

1. Introduction. The present paper is motivated by the fact that, in general, the theory and the construction of solutions of boundary value problems are more difficult than those of initial value problems [4, page 629], and by the need for a simple criterion to determine whether a boundary value problem is well posed [5, page 43].

Our main objective in this research is to establish an easy-to-apply criterion for the fundamental question of existence and uniqueness of solutions of a discrete boundary value problem (DBVP). For definiteness, we consider a general $k$ th-order linear difference equation with constant coefficients given as

$$
\sum_{j=0}^{k} a_{j} y(n+j)=0, \quad n=0,1,2, \ldots, \quad a_{0} a_{k} \neq 0,
$$

subject to separable boundary conditions of the form

$$
y(i)=y_{i}, \quad i=0, \ldots, k_{1}-1, \quad y(j)=y_{j}, \quad j=N, \ldots, N+k_{2}-1,
$$

where $k_{1}, k_{2} \geq 1, k_{1}+k_{2}=k$, and $N>k_{1}$. We establish a criterion based upon the parameters of the given boundary value problem, namely, the coefficients, $k, k_{1}$, and $N$.

In the literature, in general, existence and uniqueness theorems for DBVPs provide sufficient conditions in which a Green's function plays an essential role [6, pages 243-250]. Furthermore, unlike the results established in [1, 2, 3] and 
conjectured in [7] (despite their applicability), the result established here does not require finding the zeros of the corresponding characteristic polynomial

$$
p(\lambda)=\sum_{j=0}^{k} a_{j} \lambda^{j}
$$

which may be a difficult task for higher-degree polynomials. Similar determinants (see Theorem 2.1) were used to characterize disconjugacy of linear second-order selfadjoint difference equations in [6, page 251].

This paper is organized as follows. In Section 2, the main result, Theorem 2.1 , is presented along with several preliminary results that will be needed for the lengthy proof of the result. In Section 3, a simple algorithm for applying the criterion is presented. In Section 4, an illustrative example is given. Finally, in Section 5, we conclude our research with an important remark that illustrates the applicability of the main result to a more general type of boundary conditions.

2. Existence and uniqueness criterion. Our main result in this research is the following theorem.

THEOREM 2.1. DBVP (1.1) and (1.2) has a unique solution if and only if the following $\left(N-k_{1} \times N-k_{1}\right)$ determinant

$$
\left|\begin{array}{cccc}
a_{k_{1}} & a_{k_{1}+1} & \cdots & a_{N-1} \\
a_{k_{1}-1} & a_{k_{1}} & \cdots & a_{N-2} \\
\vdots & \vdots & \ddots & \vdots \\
a_{2 k_{1}-N+1} & a_{2 k_{1}-N+2} & \cdots & a_{k_{1}}
\end{array}\right| \neq 0
$$

where $a_{j}=0$ if $j<0$ or $j>k$.

To prove Theorem 2.1, we need to recall the following two results which were established in [1].

THEOREM 2.2. If $k, m_{1}, \ldots, m_{r}$ and $n_{1}, \ldots, n_{k}$ are positive integers such that $k=m_{1}+\cdots+m_{r}, 0 \leq n_{1}<\cdots<n_{k}, \vec{u}(z)=\left(1, z, \ldots, z^{k-1}\right)^{t}$, and $A=$ $\left(A_{1} \cdots A_{r}\right)$, where

$$
A_{j}=\left(\vec{u}\left(z_{j}\right), \ldots, \vec{u}^{\left(m_{j}-1\right)}\left(z_{j}\right)\right)
$$

and $\vec{u}^{(s)}$ is the sth componentwise derivative of the vector $\vec{u}$, then

$$
|A|=\left(\prod_{i=1}^{r} \prod_{j_{i}=0}^{m_{i}-1} j_{i} !\right) \prod_{j=2}^{r} \prod_{i=1}^{j-1}\left(z_{j}-z_{i}\right)^{m_{i} m_{j}}
$$


THEOREM 2.3. If $k, m_{1}, \ldots, m_{r}$ and $n_{1}, \ldots, n_{k}$ are positive integers such that $m_{1}+m_{2}+\cdots+m_{r}=k, 0 \leq n_{1}<\cdots<n_{k}, \vec{u}(z)=\left(z^{n_{1}}, z^{n_{2}}, \ldots, z^{n_{k}}\right)^{t}, \vec{v}_{i}(z)=$ $\left(n_{1}^{i-1} z^{n_{1}}, n_{2}^{i-1} z^{n_{2}}, \ldots, n_{k}^{i-1} z^{n_{k}}\right)^{t}$, and $B=\left(B_{1} \cdots B_{r}\right)$ such that

$$
B_{j}=\left(\vec{v}_{1}\left(z_{j}\right) \cdots \vec{v}_{m_{j}}\left(z_{j}\right)\right)
$$

then

$$
|B|=\left(\prod_{j=1}^{r} z_{j}^{m_{j}\left(m_{j}-1\right) / 2}\right)|A|,
$$

where $A=\left(A_{1} \cdots A_{r}\right)$ and

$$
A_{j}=\left(\vec{u}\left(z_{j}\right), \ldots, \vec{u}^{\left(m_{j}-1\right)}\left(z_{j}\right)\right) .
$$

Also, we need to establish the following two lemmas. The first lemma establishes a formula for higher-order derivatives of determinants. The second one plays the major role in the proof of Theorem 2.1.

LEMmA 2.4. If $F(z)=\left|C_{1}(z) \cdots C_{k}(z)\right|$ such that $C_{j}(z): \mathbb{C} \rightarrow \mathbb{C}^{k}$ are sufficiently differentiable functions, then

$$
F^{(r)}(z)=\sum_{\alpha_{1}+\cdots+\alpha_{k}=r} \frac{r !}{\alpha_{1} ! \cdots \alpha_{k} !}\left|C_{1}^{\left(\alpha_{1}\right)}(z) \cdots C_{k}^{\left(\alpha_{k}\right)}(z)\right|,
$$

where $\alpha_{i} \in\{0,1, \ldots, r\}$.

PRoof. Clearly, the result is true for $r=1$. Furthermore, if it holds for $r$, then

$$
\begin{aligned}
F^{(r+1)}= & \sum_{\alpha_{1}+\cdots+\alpha_{2}=r} \frac{r !}{\alpha_{1} ! \cdots \alpha_{k} !}\left[\left|C_{1}^{\left(\alpha_{1}+1\right)}(z) \cdots C_{k}^{\left(\alpha_{k}\right)}(z)\right|+\cdots\right. \\
& \left.+\left|C_{1}^{\left(\alpha_{1}\right)}(z) \cdots C_{k}^{\left(\alpha_{k}+1\right)}\right|\right] \\
= & \sum_{\beta_{1}+\cdots+\beta_{2}=r+1}\left[\frac{r !}{\left(\beta_{1}-1\right) ! \cdots \beta_{k} !}+\cdots\right. \\
& \left.\quad+\frac{r !}{\left(\beta_{1}\right) ! \cdots\left(\beta_{k}-1\right) !}\right]\left|C_{1}^{\left(\beta_{1}\right)}(z) \cdots C_{k}^{\left(\beta_{k}\right)}(z)\right| \\
= & \sum_{\beta_{1}+\cdots+\beta_{2}=r+1}\left[\frac{\left(\beta_{1}+\cdots+\beta_{k}\right) r !}{\beta_{1} ! \cdots \beta_{k} !}\right]\left|C_{1}^{\left(\beta_{1}\right)}(z) \cdots C_{k}^{\left(\beta_{k}\right)}(z)\right| \\
= & \sum_{\beta_{1}+\cdots+\beta_{2}=r+1} \frac{(r+1) !}{\beta_{1} ! \cdots \beta_{k} !}\left|C_{1}^{\left(\beta_{1}\right)}(z) \cdots C_{k}^{\left(\beta_{k}\right)}(z)\right| .
\end{aligned}
$$

Hence, by the principle of mathematical induction, the result is true for all $r$. 
LEMmA 2.5. Suppose that the kth-degree polynomial with complex coefficients

$$
p(\lambda)=\lambda^{k}+a_{k-1} \lambda^{k-1}+\cdots+a_{1} \lambda+a_{0}, \quad a_{0} \neq 0,
$$

has $r$ distinct roots $z_{1}, \ldots, z_{r}$ with multiplicities $m_{1}, \ldots, m_{r}$, respectively, such that $m_{1}+\cdots+m_{r}=k$.

If

$$
\begin{gathered}
E_{k}\left(\ell, m ; z_{1}, \ldots, z_{r}\right)=\left|\vec{u}_{m}\left(z_{1}\right) \cdots \vec{u}_{m}^{\left(m_{1}-1\right)}\left(z_{1}\right) \cdots \vec{u}_{m}\left(z_{r}\right) \cdots \vec{u}_{m}^{\left(m_{r}-1\right)}\left(z_{r}\right)\right|, \\
0 \leq \ell \leq k, m \geq 0
\end{gathered}
$$

where $\vec{u}_{m}(z)=\left(1, \ldots, z^{\ell-1}, z^{\ell+m}, \ldots, z^{k+m-1}\right)^{t} \in \mathbb{C}^{k}$, and

$$
D_{k}^{a}(\ell, m)=\left|\begin{array}{cccc}
a_{\ell} & a_{\ell+1} & \cdots & a_{\ell+m-1} \\
a_{\ell-1} & a_{\ell} & \cdots & a_{\ell+m-2} \\
\vdots & \vdots & \ddots & \vdots \\
a_{\ell-m+1} & a_{\ell-m+2} & \cdots & a_{\ell}
\end{array}\right|
$$

such that $a_{j}=0$ if $j<0$ or $j>k$, then

$$
E_{k}\left(\ell, m ; z_{1}, \ldots, z_{r}\right)=(-1)^{m(k-\ell)} D_{k}^{a}(\ell, m) V_{k}\left(z_{1}, \ldots, z_{r}\right),
$$

where

$$
\begin{aligned}
V_{k}\left(z_{1}, \ldots, z_{r}\right) & =E_{k}\left(\ell, 0 ; z_{1}, \ldots, z_{r}\right) \\
& =\left(\prod_{i=1}^{r} \prod_{j_{i}=0}^{m_{i}-1} j_{i} !\right) \prod_{j=2}^{r} \prod_{i=1}^{j-1}\left(z_{j}-z_{i}\right)^{m_{i} m_{j}}
\end{aligned}
$$

Proof. The last part of the above result follows from Theorem 2.2.

First, since $z_{1}, \ldots, z_{r}$ are zeros of polynomial $p$ with multiplicities $m_{1}, \ldots, m_{r}$, respectively, we have

$$
\left(\begin{array}{c}
\vec{u}_{0}^{t}\left(z_{1}\right) \\
\vdots \\
\left(\vec{u}_{0}^{t}\right)^{\left(m_{1}-1\right)}\left(z_{1}\right) \\
\vdots \\
\vec{u}_{0}^{t}\left(z_{r}\right) \\
\vdots \\
\left(\vec{u}_{0}^{t}\right)^{\left(m_{r}-1\right)}\left(z_{r}\right)
\end{array}\right)\left(\begin{array}{c}
a_{0} \\
a_{1} \\
\vdots \\
a_{k-1}
\end{array}\right)=-\left(\begin{array}{c}
z_{1}^{k} \\
\vdots \\
\left(z_{1}^{k}\right)^{\left(m_{1}-1\right)} \\
\vdots \\
z_{r}^{k} \\
\vdots \\
\left(z_{r}^{k}\right)^{\left(m_{r}-1\right)}
\end{array}\right)
$$


Therefore, by Cramer's rule and the invariance under the transposition property of the determinants, we have

$$
E_{k}\left(\ell, 1 ; z_{1}, \ldots, z_{r}\right)=(-1)^{(k-\ell)} a_{\ell} V_{k}\left(z_{1}, \ldots, z_{r}\right),
$$

that is, the result holds for $m=1$.

Now, if the result holds for $m$, then

$$
\begin{aligned}
E_{k+1} & \left(\ell+1, m ; z_{1}, \ldots, z_{r}, z\right) \\
\quad= & (-1)^{m(k-\ell)} D_{k+1}^{b}(\ell+1, m) V_{k+1}\left(z_{1}, \ldots, z_{r}, z\right),
\end{aligned}
$$

where $b_{j}=a_{j-1}-a_{j} z$ are the coefficients of the $(k+1)$-degree polynomial $q(\lambda)=(\lambda-z) p(\lambda)$. But, by Leibnitz rule of differentiation,

$$
\begin{aligned}
& (-1)^{(k+\ell)} \ell ! E_{k}\left(\ell, m+1 ; z_{1}, \ldots, z_{r}\right) \\
& \quad=\left.\frac{\partial^{\ell} E_{k+1}\left(\ell+1, m ; z_{1}, \ldots, z_{r}, z\right)}{\partial z^{\ell}}\right|_{z=0} \\
& \quad=\left.\left.(-1)^{m(k-\ell)} \sum_{j=0}^{\ell}\left(\begin{array}{l}
\ell \\
j
\end{array}\right) \frac{\partial^{j} D_{k+1}^{b}(\ell+1, m)}{\partial z^{j}}\right|_{z=0} \cdot \frac{\partial^{\ell-j} V_{k+1}\left(z_{1}, \ldots, z_{r}, z\right)}{\partial z^{\ell-j}}\right|_{z=0} .
\end{aligned}
$$

However,

$$
\begin{aligned}
\left.\frac{\partial^{i} V_{k+1}\left(z_{1}, \ldots, z_{r}, z\right)}{\partial z^{i}}\right|_{z=0} & =(-1)^{i+k} i ! E_{k}\left(i, 1 ; z_{1}, \ldots, z_{k}\right) \\
& =i ! a_{i} V_{k}\left(z_{1}, \ldots, z_{r}\right), \\
\left.\frac{\partial^{i} D_{k+1}^{b}(\ell+1, m)}{\partial z^{i}}\right|_{z=0} & =(-1)^{i} i ! M_{m+1, m+1-i},
\end{aligned}
$$

where $M_{r s}$ is the $r s$-minor of $D_{k}^{a}(\ell, m+1)$ if $r, s \geq 0$ and 0 otherwise. Therefore, after some simplifications, we obtain

$$
\begin{aligned}
E_{k}(\ell, & \left.m+1 ; z_{1}, \ldots, z_{r}\right) \\
& =(-1)^{(m+1)(k-\ell)}\left[\sum_{j=0}^{\ell}(-1)^{j} a_{\ell-j} M_{m+1, m+1-j}\right] V_{k}\left(z_{1}, \ldots, z_{r}\right) \\
& =(-1)^{(m+1)(k-\ell)} D_{k}^{a}(\ell, m+1) V_{k}\left(z_{1}, \ldots, z_{r}\right) .
\end{aligned}
$$

and, hence, by the principle of mathematical induction, the result holds for all $m$.

To complete the proof, we need to justify (2.18) and (2.19).

Equation (2.18) can be easily checked out by differentiating the last column of $V_{k+1}\left(z_{1}, \ldots, z_{r}, z\right) i$ times, substituting $z=0$ and applying the Laplace expansion of determinants through the $(k+1)$ th column. 
As for (2.19), since $b_{j}$ are linear in $z$, by Lemma 2.4,

$$
\frac{\partial^{i} D_{k+1}^{b}(\ell+1, m)}{\partial z^{i}}=\sum_{\alpha_{1}+\cdots+\alpha_{m}=i} \frac{i !}{\alpha_{1} ! \cdots \alpha_{m} !}\left|C_{1}^{\left(\alpha_{1}\right)}(z) \cdots C_{m}^{\left(\alpha_{m}\right)}(z)\right|
$$

where $\alpha_{i} \in\{0,1\}$ and $C_{j}(z)=\left(b_{\ell+j} \cdots b_{\ell+j-m+1}\right)^{t}$. But at $z=0$ and $b_{j}=a_{j-1}$,

$$
\left.\frac{\partial^{i} D_{k+1}^{b}(\ell+1, m)}{\partial z^{i}}\right|_{z=0}=i !\left|C_{1}(0) \cdots C_{m-i}(0) C_{m-i+1}^{\prime}(0) \cdots C_{m}^{\prime}(0)\right|
$$

Finally, using the fact that $b_{j}^{\prime}=-a_{j}$, the result follows. This completes the proof.

Proof of Theorem 2.1. First, without loss of generality, we assume that $a_{k}=1$.

Now, it is not difficult to see that if the characteristic polynomial (1.3) has $r$ distinct characteristic roots denoted by $z_{1}, z_{2}, \ldots, z_{r}$ with corresponding multiplicities $m_{1}, \ldots, m_{r}$ such that $1 \leq r \leq k, 1 \leq m_{j} \leq k, j=1, \ldots, r$, and $m_{1}+$ $\cdots+m_{r}=k$, then the general solution of (1.1) is given by

$$
y(n)=\sum_{i=1}^{r} q_{i}(n) z_{i}^{n}
$$

where $q_{i}(n)$ is a polynomial in $n$ of degree $m_{i}-1$. Therefore, the existence of a unique solution of the DBVP (1.1) and (1.2) is equivalent to the nonsingularity of the block matrix $A$ defined by

$$
A=\left(A_{1} \cdots A_{r}\right)
$$

where $A_{j}$ is a $k \times m_{j}$ matrix given by

$$
A_{j}=\left(\begin{array}{cccc}
1 & 0 & \cdots & 0 \\
\vdots & \vdots & & \vdots \\
z_{j}^{k_{1}-1} & \left(k_{1}-1\right) z_{j}^{k_{1}-1} & \cdots & \left(k_{1}-1\right)^{m_{j}-1} z_{j}^{k_{1}-1} \\
z_{j}^{N} & N z_{j}^{N} & \cdots & N^{m_{j}-1} z_{j}^{N} \\
\vdots & \vdots & & \vdots \\
z_{j}^{N+k_{2}-1} & \left(N+k_{2}-1\right) z_{j}^{N+k_{2}-1} & \cdots & \left(N+k_{2}-1\right)^{m_{j}-1} z_{j}^{N+k_{2}-1}
\end{array}\right)
$$

for $j=1, \ldots, r$. 
Hence, by Theorem 2.3,

$$
|A|=\left(\prod_{j=1}^{r} z_{j}^{m_{j}\left(m_{j}-1\right) / 2}\right) E_{k}\left(k_{1}, N-k_{1} ; z_{1}, \ldots, z_{r}\right) .
$$

Therefore, by Lemma 2.5, $A$ is nonsingular if and only if $D_{k}^{a}\left(k_{1}, N-k_{1}\right) \neq 0$, and so the result follows.

3. A computational algorithm. To determine the solvability of the DBVP (1.1) and (1.2), we propose the following easy-to-apply algorithm.

\section{AlgORITHM 3.1}

Input. $k$ (order of the difference equation), $a_{i}, i=0,1, \ldots, k$ (coefficients), $k_{1}$ (number of initial conditions), $N$ (starting index of final conditions).

Step 1. Form the following table:

$$
\begin{array}{cccccccc}
a_{0} & a_{1} & a_{2} & \cdots & a_{k} & 0 & 0 & \cdots \\
0 & a_{0} & a_{1} & \cdots & a_{k-1} & a_{k} & 0 & \cdots \\
0 & 0 & a_{0} & \cdots & a_{k-2} & a_{k-1} & a_{k} & \cdots \\
\vdots & \vdots & \vdots & \ddots & \vdots & \vdots & \vdots & \ddots
\end{array}
$$

Step 2. Skip $k_{1}$ columns by starting from the left.

Step 3. Form a determinant of size $\left(N-k_{1}\right) \times\left(N-k_{1}\right)$ starting from the first row.

Output. If the determinant calculated in Step 3 is not zero, then DBVP (1.1) and (1.2) has a unique solution.

REMARK 3.2. It is worthwhile mentioning that the determinant needed in the above algorithm corresponds to a banded matrix with constant diagonals. In fact, there will be at most $k+1$ diagonals. This will definitely minimize the cost of computations as far as computer time is concerned.

4. Applications. To illustrate the applicability of Theorem 2.1 (and Algorithm 3.1), we present the following example.

EXAMPLE 4.1. Consider the difference equation

$$
y(n+k)+a y(n)=0, \quad a \neq 0, n=0,1,2, \ldots
$$

subject to the boundary conditions

$$
y(0)=y_{0}, \quad y(N+1)=y_{N+1}, \ldots, y(N+k-2)=y_{N+k-2}, \quad N \geq 1 .
$$

By Theorem 2.1, if $1 \leq N \leq k-1$, then a solution of the DBVP (4.1) and (4.2), if it exists, is not unique. Furthermore, for $N \geq k$, it is not difficult to see that 
a unique solution exists if and only if the $N \times N$ determinant

$$
D_{N}(a)=\left|\begin{array}{ccccc}
0 & & 1 & & \\
a & 0 & & 1 & \\
& \ddots & \ddots & & \ddots \\
& & a & 0 &
\end{array}\right| \neq 0
$$

where the missing elements are zeros.

But

$$
D_{N}(a)=(-a)^{k-1} D_{N-k}(a), \quad N=k, k+1, \ldots,
$$

where

$$
D_{0}=1, \quad D_{1}=\cdots=D_{k-1}=0 .
$$

Therefore, one can see that $D_{n}=0$ for all $N$ except for $N=0, k, 2 k, \ldots$. Hence, the DBVP (4.1) and (4.2) has a unique solution if and only if $N=0, k, 2 k, \ldots$ Otherwise, if there is a solution, it is not unique.

5. Conclusion. Finally, it is important to mention that Theorem 2.1 is also applicable for a wider class of boundary conditions. Namely, the boundary conditions are described as

$$
B\left(\begin{array}{c}
y(0) \\
\vdots \\
y\left(k_{1}-1\right) \\
y(N) \\
\vdots \\
y\left(N+k_{2}-1\right)
\end{array}\right)=\vec{y}_{b},
$$

in which $B$ is a $k \times k$ matrix of full rank.

ACKNOWLEDGment. The authors would like to extend their thanks to the University of Sharjah for financial support.

\section{REFERENCES}

[1] R. Abu-Saris, Two point discrete boundary value problems, submitted to Journal of Computational Analysis and Applications.

[2] R. Abu-Saris and Kh. Al-Dosary, Complex exponential Vandermonde determinant with application to discrete boundary value problem, Proceedings of the 7th International Conference on Difference Equations and Applications, Field Institute Communications, to appear.

[3] R. Abu-Saris and H. Yousef, Existence theorems for boundary value problems in difference equations, J. Differ. Equations Appl. 7 (2001), no. 2, 255-263. 
[4] R. P. Agarwal, Difference Equations and Inequalities: Theory, Methods, and Applications, 2nd ed., Monographs and Textbooks in Pure and Applied Mathematics, vol. 228, Marcel Dekker, New York, 2000.

[5] C. M. Bender and S. A. Orszag, Advanced Mathematical Methods for Scientists and Engineers. I. Asymptotic Methods and Perturbation Theory, Springer-Verlag, New York, 1999.

[6] W. G. Kelley and A. C. Peterson, Difference Equations. An Introduction with Applications, 2nd ed., Harcourt/Academic Press, California, 2001.

[7] G. Ladas, Open problems and conjectures, J. Differ. Equations Appl. 4 (1998), 395396.

Raghib Abu-Saris: Department of Basic Sciences, University of Sharjah, P.O. Box 27272, Sharjah, United Arab Emirates

E-mail address: rabusaris@sharjah.ac.ae

Wajdi Ahmad: Department of Electrical and Electronics Engineering, University of Sharjah, P.O. Box 27272, Sharjah, United Arab Emirates

E-mail address: wajdi@sharjah.ac.ae 


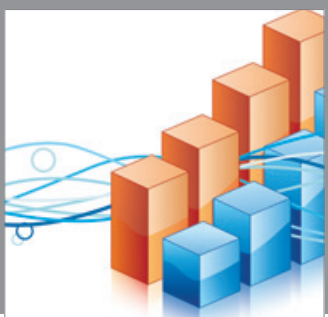

Advances in

Operations Research

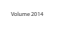

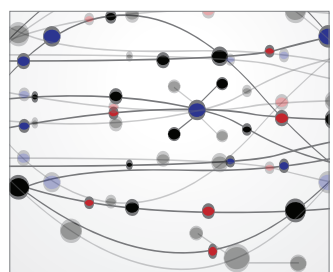

\section{The Scientific} World Journal
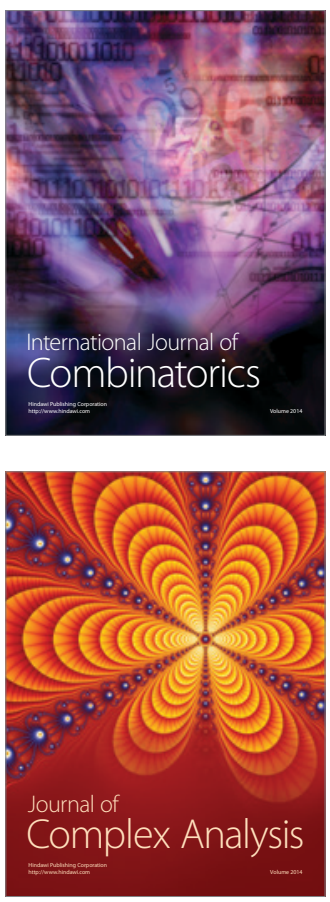

International Journal of

Mathematics and

Mathematical

Sciences
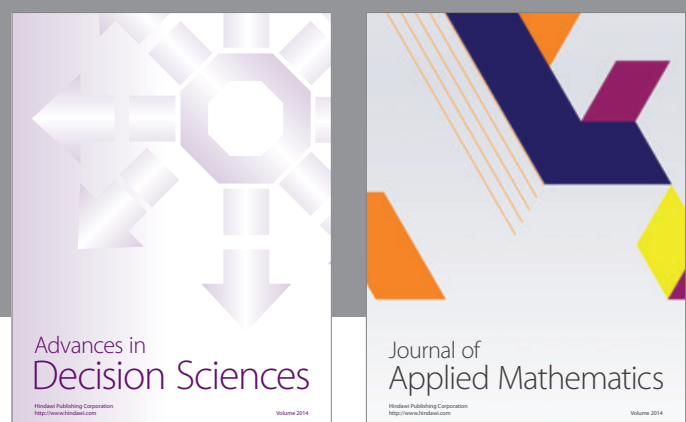

Journal of

Applied Mathematics
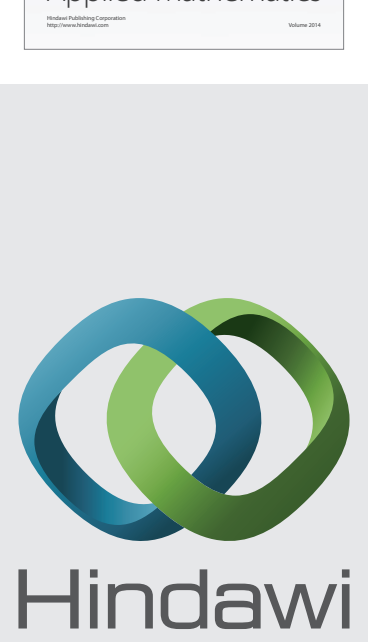

Submit your manuscripts at http://www.hindawi.com
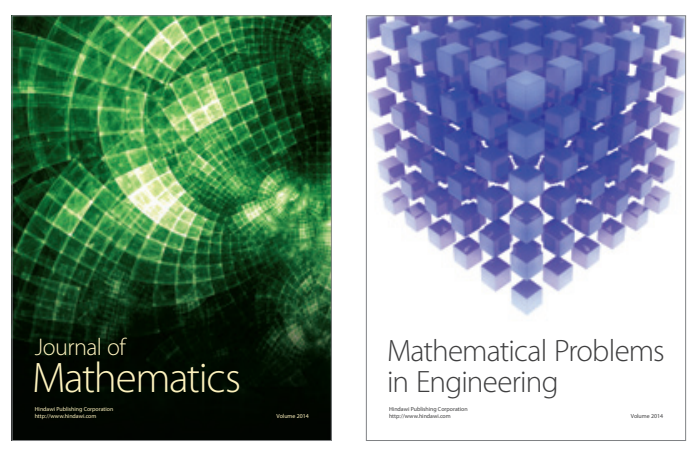

Mathematical Problems in Engineering
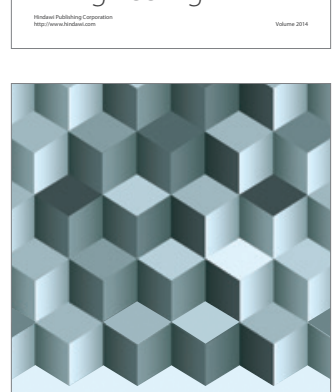

Journal of

Function Spaces
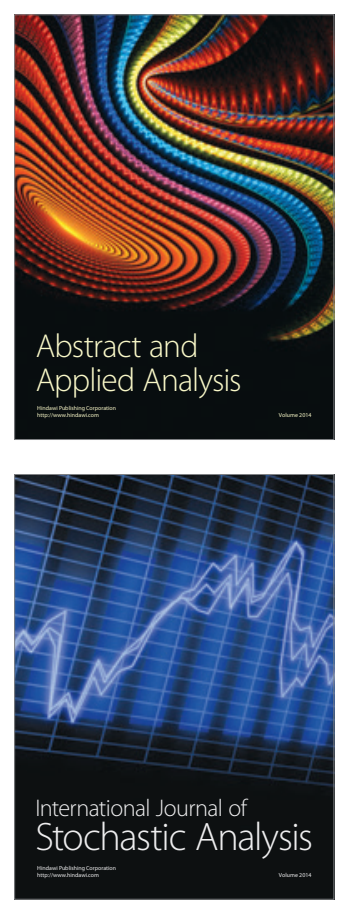

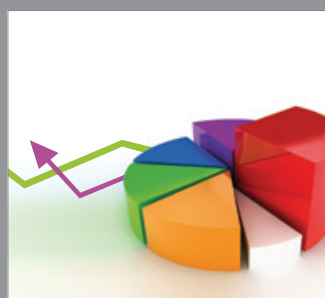

ournal of

Probability and Statistics

Promensencen
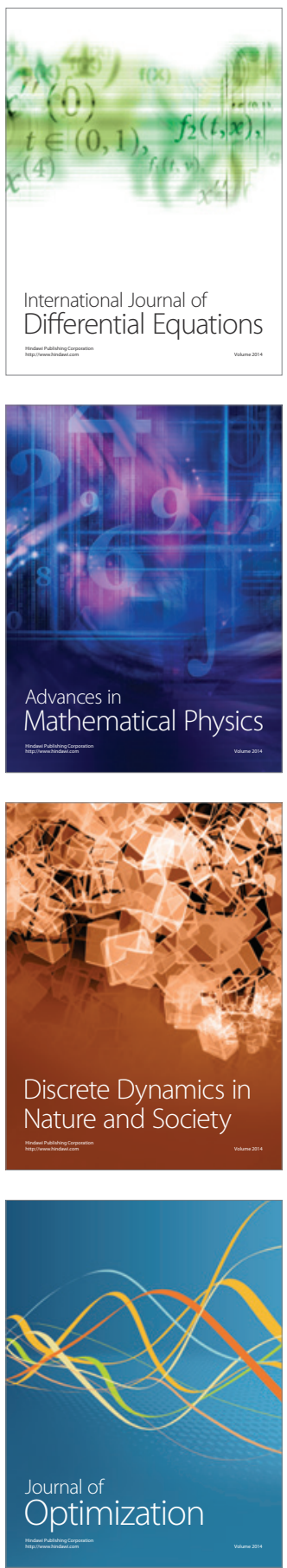